\title{
Reposicionando la cloxacilina como antibioticoterapia empírica inicial de la sepsis tardía neonatal
}

\author{
Alejandra Sandoval, Fernanda Cofré, Luis Delpiano, Giannina Izquierdo, Yenis Labraña y Alejandra Reyes
}

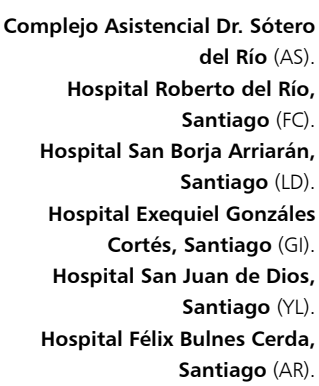

Integrantes del Comité Consultivo de Infecciones Neonatales de la Sociedad Chilena de Infectología.

Fuente de financiamiento: ninguno.

Conflictos de interés: Sin conflictos de interés por parte de los autores.

Recibido: 18 de noviembre de 2014 Aceptado: 10 de marzo de 2015

Correspondencia a: Alejandra Sandoval C ale7sandov@gmail.com

\section{Introducción}

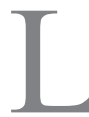

os recién nacidos $(\mathrm{RN})$, y en particular los prematuros de muy bajo peso de nacimiento (RNPret MBPN), definidos como todos aquellos prematuros menores de $1.500 \mathrm{~g}$ ó menores de 32 semanas, tienen un sistema inmunitario inmaduro y son altamente susceptibles a las infecciones por microorganismos grampositivos durante su estadía en una Unidad de Cuidados Intensivos Neonatal (UCIN) ${ }^{1}$.

Agentes como Staphylococcus coagulasa negativa (SCN) son los causantes más comunes de sepsis neonatal de inicio tardío en las UCIN; sin embargo, su virulencia es baja y la sepsis fulminante es inusual, ocurriendo sólo en $1 \%$ de los $\operatorname{casos}^{2-4}$. En Latinoamérica, la escasa literatura científica publicada al respecto coincide en que el microorganismo más frecuente de sepsis neonatal tardía es SCN, fluctuando con incidencias entre 29 y $50 \%$; esta gran variabilidad es reflejo de la epidemiología local de cada centro en los distintos países con registros publicados $^{5-7}$. En el informe de Infecciones Asociadas a la Atención de Salud (IAAS) en Chile entregado por el Ministerio de Salud (MINSAL) para el año 2012, se reportaron 28 infecciones del tracto sanguíneo (ITS) en neonatos con catéter umbilical, con una tasa de $2,24 \mathrm{x}$ 1.000 días de exposición, siendo identificado en 33,3\% de los casos de $\mathrm{SCN}^{8}$.

Pese a la baja virulencia de estos microorganismos, el aumento de la resistencia a antimicrobianos $\beta$-lactámicos, ha llevado a que en muchos países se utilice empíricamente vancomicina como antibacteriano de elección para el manejo inicial de la sepsis tardía en $\mathrm{UCIN}^{3,9}$.

A pesar de que vancomicina se utiliza desde hace más de 50 años, su prescripción sigue siendo un problema en las UCIN por diversas razones, incluyendo: alta variabilidad farmacocinética y con ello, dificultad para alcanzar concentraciones plasmáticas óptimas, falta de consenso sobre el régimen de dosificación y monitorización terapéutica, aumento de la concentración inhibitoria mínima (CIM) que implica una mayor probabilidad de seleccionar cepas resistentes ${ }^{9}$ y aumento de otras infecciones en las UCIN.

En el marco del uso racional de antimicrobianos antiestafilocóccicos y considerando lo anteriormente señalado, nos ha parecido de especial interés revisar los fundamentos teóricos en que se basan estos cambios y realizar una reseña acerca de las características clínico-farmacológicas de los antimicrobianos en la población neonatal, que son distintas a las de la población pediátrica en general.

\section{Comportamiento farmacológico de los antimicrobianos en los neonatos}

Los rápidos cambios en los procesos fisiológicos del desarrollo fetal y neonatal afectan profundamente las propiedades farmacocinéticas $(\mathrm{FK})$ y farmacodinámicas (FD) de los antimicrobianos; el proceso de maduración de los diversos sistemas en el RN influye directamente en la distribución del agua corporal, en el metabolismo 
y en la eliminación de todos los fármacos, lo que, potencialmente puede llevar a la obtención de concentraciones sub-terapéuticas de los mismos ${ }^{10}$.

El volumen de distribución en el RNPret suele ser mayor que en los recién nacidos de término (RNT), debido a que el compartimento de agua corporal es mayor a menor edad gestacional, afectando directamente la concentración de los antimicrobianos, especialmente aquellos hidrosolubles, los que, al distribuirse en un mayor volumen corporal generan concentraciones séricas menores y una vida media más prolongada ${ }^{10}$.

La unión a proteínas plasmáticas también puede afectar la distribución y eliminación de los fármacos. Las variables que influyen en la unión a proteínas incluyen las concentraciones de albúmina, concentración del fármaco, afinidad de los antimicrobianos en la unión a proteínas y presencia de fármacos ó metabolitos que compiten por los sitios de unión a proteínas por ejemplo: furosemida, bilirrubina, etc ${ }^{10}$.

La nefrogénesis comienza a las 5-6 semanas de gestación y continúa hasta las 36 semanas, de tal forma que, otra variable a considerar es la función renal, la cual varía con la edad gestacional, edad cronológica y condición clínica $^{10,11}$. En los primeros cinco días de vida los valores de creatinina sérica reflejan la función renal materna. Sin embargo, durante las primeras semanas de vida existe un rápido aumento de la tasa de filtración glomerular (TFG), dado principalmente por los cambios hemodinámicos y la disminución en la resistencia vascular ${ }^{10}$. En los RN en general, la TFG es 30 a 60\% de la del adulto; no obstante, como existe un aumento notable en la función renal durante las dos primeras semanas de vida, la depuración de medicamentos es mayor en los RN y lactantes en comparación con los niños mayores y adultos ${ }^{10}$.

Distinta es la situación de los RNPret, ya que, después del nacimiento tienen una función renal que mejora más lentamente, llevando a la eliminación prolongada de los fármacos durante las primeras semanas de vida. La eliminación del fármaco también se reduce en los RNPret gravemente enfermos debido a la disminución del flujo sanguíneo renal como consecuencia de la insuficiencia respiratoria, hipotensión arterial o deshidratación. Es importante definir las dosis y frecuencia de la administración de antimicrobianos en este grupo de pacientes dado que la función renal está en constante cambio durante el primer mes de $\operatorname{vida}^{10}$.

\section{Características de dos de los principales antimicrobianos antiestafilocóccicos utilizados en neonatología}

\section{Vancomicina}

Vancomicina tiene acción bactericida frente a las cocáceas grampositivas. Las infecciones causadas por
Staphylococcus aureus resistente a meticilina (SARM) y Enterococcus resistentes a ampicilina constituyen las principales indicaciones del uso de vancomicina en $\mathrm{RN}^{10}$.

La vancomicina no se metaboliza, se une en $55 \%$ a proteínas plasmáticas y se elimina principalmente, sin cambios, en la orina por filtración glomerular ${ }^{10,12}$. La farmacocinética de vancomicina se ve afectada de manera significativa por los cambios fisiológicos que se producen durante el período neonatal. La eliminación de vancomicina aumenta con la edad gestacional y cronológica, lo que refleja la maduración renal. El volumen de distribución varía con el peso y es mayor en los RNPret y RN que lo reportado en niños mayores ${ }^{10,12}$. El peso, la edad gestacional y el volumen de distribución influyen además, directamente sobre la función renal y teniendo en cuenta que, en neonatos la función renal es inmadura, van a determinar una depuración reducida de los antimicrobianos contribuyendo a una vida media más prolongada, lo cual debe considerarse al momento de determinar la dosis óptima de vancomicina en neonatos ${ }^{10,12}$. Factores tales como: persistencia del conducto arterioso, el uso de indometacina o ibuprofeno y la oxigenación por membrana extracorpórea (en inglés ECMO), también influyen en el clearance de medicamentos ${ }^{12}$.

\section{Concentraciones plasmáticas}

En relación a concentraciones terapéuticas, el rango objetivo para los niveles basales debieran ser al menos entre 10 y $15 \mu \mathrm{g} / \mathrm{mL}$, ya que, con concentraciones de $12 \mu \mathrm{g} / \mathrm{mL}$ se han asociado a curas clínicas en niños ${ }^{10}$. Hay aparición progresiva de literatura científica que plantea como concentración terapéutica objetivo en neonatos valores de 20-25 $\mu \mathrm{g} / \mathrm{L}$, especialmente en los pacientes críticos. Sin embargo, en la población neonatal este rango se alcanza sólo en $46 \%$ de los pacientes y hasta en un 20 $25 \%$ las concentraciones son menores a $10 \mu \mathrm{g} / \mathrm{mL}^{12,13}$. La imposibilidad de alcanzar concentraciones óptimas lleva a reajustar constantemente las dosis alcanzando incluso dosis de hasta $80 \mathrm{mg} / \mathrm{kg} /$ día. Este problema se debe entre otras cosas a la diferencia inter-individual de esta población en cuanto a sus volúmenes de distribución y al creciente aumento de la CIM de vancomicina ${ }^{13}$, que puede llegar a ser $2 \mu \mathrm{g} / \mathrm{ml}$ para SCN. Lo anterior ha generado una tendencia a utilizar protocolos de infusión continua o prolongadas de vancomicina basados en el peso, edad gestacional corregida y creatininemia. Las ventajas del uso de la infusión continua o prolongada es que se logran concentraciones plasmáticas más estables y óptimas ${ }^{13}$; sin embargo, existen pocos estudios en población neonatal y por ahora en la práctica clínica es poco realizable, debido al bajo peso de estos pacientes y a las múltiples infusiones que reciben en forma simultánea ${ }^{12,14}$.

En base a lo expuesto sobre las concentraciones plas- 
máticas terapéuticas, lamentablemente faltan estudios para definir la forma óptima de realizar el cálculo de la dosificación de vancomicina en neonatos ${ }^{12}$. Por ahora hay creciente literatura científica que promueve la dosificación de vancomicina basada en la creatininemia, ya que, se ha visto que la utilización de este parámetro para el cálculo de las dosis neonatales es bastante confiable y útil para la aplicación clínica debido a la facilidad en su uso ${ }^{15,16}$. Otras variables clásicas que se consideran para el cálculo de la dosis como el peso de nacimiento, peso actual y la edad postnatal, lamentablemente conducen a cálculos complejos, tanto para la dosis de carga como para la de mantención ${ }^{17}$.

La implementación de un esquema óptimo de dosificación de vancomicina para RNPret permitiría mejorar la eficiencia del tratamiento, reducir la necesidad de monitorización y obtener concentraciones séricas de vancomicina potencialmente más efectivas y seguras ${ }^{18}$. Es así que, surge la urgencia de realizar una validación de los regímenes de dosificación, en base a estudios FK y FD prospectivos en $\mathrm{RN}^{19}$ y la necesidad de contar con más evidencia científica que evalúe la eficacia bacteriológica de vancomicina administrada en infusión continua o prolongada, ya que, representa un nuevo enfoque que merece la realización de estudios prospectivos, controlados, con especial atención en la toxicidad y en la eficacia terapéutica en los neonatos ${ }^{20,21}$. Sin duda, el reto de las infecciones neonatales nosocomiales por Staphylococcus spp merece intentos cuidadosos que permitan optimizar su tratamiento y el diseño de estos estudios debe considerar un conocimiento exhaustivo de la fisiología renal y la farmacología en el neonato ${ }^{21}$.

\section{FK/FD, CIM e implicaciones clínicas para la dosificación de vancomicina}

Farmacocinética: La heterogeneidad FK de vancomicina en RN y lactantes plantea un reto importante para la optimización de las dosis de este antimicrobiano y, a evitar el uso de dosis estándar sin monitorización de dosis terapéutica.

Los RN tienen un mayor porcentaje de agua corporal y experimentan rápidos cambios postnatales en la eliminación y depuración de los medicamentos asociados al funcionamiento renal dentro de la primera semana de vida $^{11}$. Estudios del perfil FK de vancomicina en RNT y RNPret han demostrado un aumento de su eliminación a mayor edad gestacional y cronológica, lo que se traduce en una disminución de la vida media de vancomicina ${ }^{10}$. La vida media disminuye de $7 \mathrm{~h}$ en la primera semana de vida hasta alcanzar $4 \mathrm{~h}$ en el lactante. En el caso de los RNPret, la vida media de vancomicina es prolongada y variable e inversamente proporcional al peso, es así como, en el primer mes de vida en el neonato bajo $1.000 \mathrm{~g}$ llega aproximadamente a $10 \mathrm{~h}$, lo que significa que el tiempo para alcanzar el estado de equilibrio podría ser de hasta $50 \mathrm{~h}^{10,12}$.

En el mayor estudio de FK poblacional efectuado en 374 RN y niños bajo 2 años de edad, en el cual se midieron las concentraciones de vancomicina, se pudo establecer que la creatininemia estuvo fuertemente relacionada con la eliminación de vancomicina, siendo la edad cronológica y la prematuridad ( $<28$ semanas) predictores menos importantes que el clearance renal de vancomicina ${ }^{22}$.

Sin embargo, aun cuando las variables más importantes (edad, peso y clearence de creatinina) son consideradas en los RN, la variabilidad inter-individual aún es elevada, siendo comparativamente entre los RNPret y RNT de $23 \%$ para el clearence de vancomicina y de $47 \%$ para el volumen de distribución ${ }^{23}$. Anderson y cols, comunicaron en RNPret que la mayor parte de la variabilidad en el clearence de vancomicina se explica por la diferencia en el peso (49\%), edad postnatal (18\%) y la función renal $(14 \%)^{23}$. Por lo tanto, los intentos de comprender la variabilidad FK de vancomicina en neonatos y niños pequeños generalmente se basan en métodos que tratan de cuantificar la depuración renal de vancomicina ${ }^{23}$.

Como se mencionó previamente, debido a que la creatininemia del RN no refleja la función renal endógena sino la materna, este parámetro debe considerarse en las estimaciones de dosis individuales y poblacionales ${ }^{23}$. Además, en los neonatos se encuentra disminuida la unión de los fármacos a las proteínas, debido a dos situaciones, una es que las concentraciones de albúmina son inferiores y la segunda es que la albúmina fetal tiene una afinidad de unión inferior a los medicamentos ${ }^{23}$.

En general, los modelos FK todavía contienen suficientes niveles de variabilidad inter-individual no explicada, lo que justifica la monitorización de dosis terapéutica para el ajuste de dosis y así con ello alcanzar una concentración FD objetivo $^{23}$.

Farmacodinamia: Se ha observado en infecciones por SARM, que el parámetro FD que mejor predice la eficacia de vancomicina es el área bajo la curva por sobre la concentración inhibitoria mínima (AUC/CIM) la cual debe ser $>400 \mathrm{mg} * \mathrm{~h} / \mathrm{L}$; actualmente la monitorización terapéutica se centra en este parámetro para optimizar los objetivos de eficacia FK/FD y lograr el éxito clínico y microbiológico del tratamiento antimicrobiano ${ }^{23}$. Lo anterior explica que vancomicina actúe principalmente dependiendo del tiempo sobre la CIM, pero algo más independiente de la concentración, teniendo además moderado efecto post-antibiótico ${ }^{10}$. Estudios recientes, incluyendo niños a partir de 3 meses, han determinado que para alcanzar AUC/CIM $>400 \mathrm{mg} * \mathrm{~h} / \mathrm{L}$, se requieren concentraciones séricas mínimas de aproximadamente $7-10 \mu \mathrm{g} / \mathrm{mL}$ y una $\mathrm{CIM} \leq 1 \mu \mathrm{g} / \mathrm{ml}^{23}$; sin embargo, este resultado aún no se ha confirmado en los $\mathrm{RN}$ y hasta 
ahora la mayoría de los datos existentes corresponden a extrapolación de la población adulta y el objetivo FD más adecuado sigue siendo motivo de debate ${ }^{23}$.

Tanto el aumento de AUC/CIM y el mantener el nivel valle por mayor tiempo sobre la CIM, han demostrado que promueven la eliminación bacteriana y son condiciones necesarias para que el tratamiento de vancomicina sea eficaz ${ }^{10}$. Esta eliminación bacteriana, producto de la supresión continua del crecimiento bacteriano puede persistir durante varias horas, siendo de 0,6-2 h para $S$. aureus y de 4-6 h para SCN. SARM y SCN son normalmente sensibles a vancomicina con CIM $<2 \mu \mathrm{g} / \mathrm{ml}$; no obstante, datos recientes en neonatos muestran que la eficacia de vancomicina contra Staphylococcus (principalmente $S$. aureus), se reduce cuando existe una CIM a vancomicina de $1,5-2 \mu \mathrm{g} / \mathrm{ml}$ o superior, con aumento consiguiente de las tasas de fracaso del tratamiento frente a esta infección ${ }^{10}$.

Acuña y cols. demostraron que en pacientes pediátricos con valores basales de vancomicina entre 10 y $15 \mu \mathrm{g} / \mathrm{mL}$, $81 \%$ de ellos alcanza valores de AUC $24 \mathrm{~h} / \mathrm{CIM}>400$ $\mathrm{mg} * \mathrm{~h} / \mathrm{L}$ y la concentración plasmática pico de vancomicina fue importante en las determinaciones de AUC $24 \mathrm{~h} /$ $\mathrm{CIM}>400 \mathrm{mg} * \mathrm{~h} / \mathrm{L}$, demostrándose una correlación lineal en los niños bajo los 2 años de edad ${ }^{24}$.

Una comunicación nacional de Torres y cols, en vías de publicación, realizado en el Hospital Luis Calvo Mackenna (Santiago, Chile) y presentado en el ICAAC 2013 incluyó 20 niños de UCIN, reclutados prospectivamente; sólo $65 \%$ alcanzaron concentraciones de vancomicina de $5-15 \mu \mathrm{g} / \mathrm{ml}$ y de estos sólo $38 \%$ obtuvieron AUC de más de $400 \mathrm{mg} * \mathrm{~h} / \mathrm{L}$, lo que permitió concluir que los neonatos ingresados en UCIN probablemente requieren mayores dosis de vancomicina para alcanzar dosis terapéuticas ${ }^{25}$.

La dosificación tradicional de vancomicina en neonatos es $10-15 \mathrm{mg} / \mathrm{kg} /$ dosis cada 12 h (20-30 mg/kg/día) en la primera semana de vida y cada $8 \mathrm{~h}(30-45 \mathrm{mg} / \mathrm{kg} /$ día $)$ posterior a ello ${ }^{26}$, y por lo cual frente a todo lo expuesto es probable que esta dosificación deba tener modificaciones, sobre todo en los RNPret MBPN y debiera decidirse en forma personalizada, caso a caso en particular, con el fin de asegurar el éxito terapéutico, dado que los regímenes de dosificaciones estándares no entregan seguridad acerca de las dosis clínicamente efectivas ${ }^{10}$. Además, otros factores a considerar son los RNPret y lactantes con insuficiencia renal y/o ECMO, ya que, probablemente necesiten un control más frecuente de concentraciones para alcanzar la eficacia terapéutica esperada ${ }^{10}$.

\section{Cloxacilina}

Es el pilar de la terapia de RN para Staphylococcus sensibles a meticilina. Su principal vía de eliminación es renal. En los RN las concentraciones séricas máximas de
50 y $100 \mu \mathrm{g} / \mathrm{ml}$ se obtienen con dosis de $20-50 \mathrm{mg} / \mathrm{kg}$ y en RNPret la vida media en suero es de aproximadamente $3 \mathrm{~h}$ en la primera semana de vida disminuyendo a $1,5 \mathrm{~h}$ posteriormente $^{10}$.

\section{FK/FD e implicaciones clínicas para la dosificación} de cloxacilina

Al igual que otros $\beta$-lactámicos, su acción es dependiente del tiempo que se mantiene el fármaco sobre la CIM, al aumentar su eliminación por la maduración renal y hepática de los RN, los intervalos de dosificación se acortan con el objetivo terapéutico de mantener la concentración plasmática sobre la CIM en $50 \%$ del intervalo entre dosis y dosis. La dosis de cloxacilina es de $25-50 \mathrm{mg} / \mathrm{kg} / \mathrm{dosis}$ cada $12 \mathrm{~h}$ (50 a $100 \mathrm{mg} / \mathrm{kg} / \mathrm{día}$ ) en la primera semana de vida y luego de la primera semana es cada 6 a 8 h ( 75 a $200 \mathrm{mg} / \mathrm{kg}$ por día). Las dosis más elevadas están indicadas para los lactantes con enfermedad estafilocóccica diseminada o meningitis. Como su FK/FD es conocido y estable, sólo con variaciones de la dosificación por edad gestacional y peso de nacimiento, es posible alcanzar fácilmente el objetivo terapéutico que asegure la eficacia clínica ${ }^{10}$.

\section{Seguridad}

Las penicilinas anti-estafilocóccicas administradas por vía parenteral son bien toleradas en los RN; sin embargo, no se recomienda su administración por vía intramuscular repetida dado que puede causar daño muscular, absceso estéril del músculo y elevación de la creatininemia. La nefrotoxicidad se manifiesta como nefritis intersticial, la cual es rara en $\mathrm{RN}$, pero ocurre en $3-5 \%$ de los niños que reciben altas dosis del antimicrobiano. Alteraciones hematológicas como neutropenia reversible o eosinofilia son de incidencia desconocida en los $\mathrm{RN}^{10}$.

\section{¿Por qué no se aconseja el uso empírico de vancomicina como terapia inicial en sepsis tardías en UCIN?}

\section{Por eventuales efectos adversos}

Nefrotoxicidad: El mecanismo inducido por vancomicina para generar el daño renal no está bien establecido y como en muchas condiciones tóxicas, la nefrotoxicidad estaría relacionada con el daño oxidativo sobre las células renales tubulares proximales. Este efecto puede verse favorecida por condiciones terapéuticas que se suman en esta población como son: CIM elevadas, terapia prolongada (más de 7 días), tratamiento concomitante con aminoglucósidos, anfotericina, furosemida, indometacina e ibuprofeno ${ }^{11,27}$. Estas situaciones son frecuentes debido a la gravedad de los niños en las UCIN, a la concomitancia de otras situaciones clínicas que los aquejan (ductus 
arterioso persistente) y al escaso recurso disponible que permita controlar niveles plasmáticos.

Su importancia en el contexto de la fisiología neonatal aún está por establecerse. Al igual que con gentamicina, la inmadurez puede brindar protección al $\mathrm{RN}$ frente a la toxicidad por vancomicina, debido al potencial glomerulogénico del RN sobre todo a edades más precoces a diferencia de los adultos. Sin embargo, la exposición a otros fármacos potencialmente nefrotóxicos durante la glomerulogénesis activa, podría poner en peligro la adquisición de glomérulos; es por ello que una correcta farmacovigilancia (seguimiento de la función renal) debiera indicarse en RNPret hasta las 35 semanas de edad gestacional corregida ${ }^{21}$.

\section{Por selección de infecciones con otros microorganismos y/o resistencias}

El aumento de la prevalencia mundial de Enterococcus resistentes a vancomicina (ERV) y la grave amenaza que representa la propagación de Staphylococcus resistentes a vancomicina ha desalentado el uso de la vancomicina para la profilaxis antimicrobiana o la terapia empírica inicial $^{10}$. Esto se ha facilitado por el uso prolongado de antimicrobianos sin la suspensión precoz luego de no comprobarse la presencia de infección, ya sea, por la ausencia de síntomas clínicos y/o hemocultivos negativos, provocando el aumento de las tasas de infecciones por microorganismos resistentes y gramnegativos. Se ha demostrado adicionalmente, que vancomicina es uno de los principales antimicrobianos que favorecen el aumento de las tasas de infecciones por candidas en una UCIN, lo que constituye un grave y emergente problema en las UCIN en el mundo con aumento significativo de la morbi-mortalidad ${ }^{28}$. La aparición de microorganismos resistentes a vancomicina, como Enterococcus y $S$. aureus constituye un problema agregado al ya expuesto, dado que pueden causar infecciones graves, potencialmente mortales, con opciones terapéuticas limitadas y de alto costo, ocasionando importantes impactos en la salud pública. Las políticas de restricción del uso de vancomicina son consideradas de gran importancia para prevenir el desarrollo y propagación de tales microorganismos, siendo recomendadas ampliamente por el Centers for Disease Control and Prevention (CDC) a través del control de IAAS y constituyendo la restricción del uso de vancomicina un parámetro de medición de calidad dentro de una unidad de paciente crítico ${ }^{3}$.

\section{Otros argumentos para restringir el uso de vancomicina}

La baja mortalidad de la sepsis por SCN en pacientes de UCIN permite retrasar el inicio de vancomicina como terapia empírica en sepsis neonatal tardía. Algunos estudios retrospectivos realizados en UCIN respaldan esta restricción, demostrando que la duración y la mortalidad de la sepsis fue similar en los grupos tratados con oxacilina más aminoglucósido comparado con vancomicina, en el tratamiento empírico de la sepsis por SCN, incluso si éstos en su mayoría son resistentes a oxacilina ${ }^{4}$. El efecto podría explicarse en parte por la interacción sinérgica de estos dos agentes antimicrobianos, apoyado en parte por algunos experimentos in vitro que lo demuestran ${ }^{4}$.

La mayoría de las UCIN no tienen directrices para restringir el uso de vancomicina; sin embargo, existen varios estudios que apoyan la seguridad de la restricción del uso de vancomicina en UCIN. Uno de ellos con diseño retrospectivo, comparó los períodos durante los cuales se utilizó oxacilina o vancomicina en el tratamiento empírico de sepsis neonatal tardía y no encontró diferencias estadísticas en cuanto a duración de la bacteriemia y mortalidad $^{29}$. Favorablemente, este estudio se realizó antes del aumento de la incidencia de colonización e infección por SARM, lo que fomentó la idea de limitar el uso de vancomicina empírica en UCIN en lugares con baja incidencia de SARM. En el 2006 se demostró que la aplicación de una guía de utilización de vancomicina, en dos UCIN con bajas prevalencias de infecciones por SARM (aproximadamente menos del 2\%), redujo de manera segura y sostenible el uso de vancomicina como primera línea en la sepsis tardía, ya que no hubo aumento de mortalidad y morbilidad (bacteriemia prolongada y meningitis), con óptimo manejo del cuadro infeccioso, demostrándose que la terapia con cloxacilina no era inferior a la vancomicina ${ }^{29}$.

\section{¿Por qué el resurgimiento de cloxacilina?}

Frente a la problemática generada por el uso de vancomicina en cuanto a la dificultad en titular dosis, concentraciones séricas sub-terapéuticas y emergencia de patógenos resistentes, se comenzaron a publicar diversos estudios, inicialmente retrospectivos, que sugirieron la restricción del uso de vancomicina, incluso en casos confirmados de sepsis por SCN. Además de que, la disminución de su uso no se asoció a un aumento de la mortalidad o morbilidad, incluso cuando las tasas de resistencia a oxacilina son altas $^{3}$, sugiriendo entonces que el uso de vancomicina empírica para la sepsis neonatal tardía no se justifica en todos los centros de UCIN, y esto dependerá básicamente de la incidencia de infecciones por SARM en cada centro. La aplicación de pautas de restricción del uso de vancomicina versus no restricción de su uso ha demostrado que el outcome duración de sepsis y mortalidad es similar con cloxacilina y vancomicina, sin demostrarse inferioridad con cloxacilina ${ }^{3}$.

En base a lo anterior y a las nuevas estrategias de restricción de antimicrobianos, existen varios grupos 
de estudio en el mundo que han tomado la decisión de utilizar cloxacilina como tratamiento de primera línea en sepsis neonatal tardía. Algunos fundamentos de ellos corresponden a lo comunicado por Karlowitz y cols. ${ }^{28,30}$, que con una experiencia de cuatro años concluyó que el uso de cloxacilina resultó más que razonable debido a la baja frecuencia de sepsis fulminante causada por SCN. Además se ha planteado que el uso de cloxacilina puede tratar con éxito la bacteriemia por SCN en hasta $89 \%$, a pesar de la resistencia in vitro de la mayoría de los aislados que se eleva incluso a $85 \%{ }^{30}$.

En relación a la resistencia antimicrobiana, la Academia Americana de Pediatría (AAP) establece que los puntos de corte de CIM de SCN para meticilina se basan en la presencia del gen mecA, y es éste el que se asocia al potencial del microorganismo para desarrollar resistencia. La presencia de este gen en SCN, sin embargo, no predice una medida exacta de la expresión de genes que determinen la susceptibilidad o resistencia ${ }^{27}$; esto se debe a que la presencia del gen mecA en este microorganismo no siempre se traduce en la producción de la PBP-2a, cuestionándose la interpretación de esta prueba in vitro; cabe eso si destacar que, esta situación no ocurre y es diferente con SARM ${ }^{30,31}$.

Tomando en cuenta que el uso de cloxacilina puede conducir a la resolución clínica y de laboratorio de la infección en la mayoría de los neonatos con sepsis por SCN y con ello se evita el uso de vancomicina en más de la mitad de los niños, sólo en aquellos casos en que no se logra la mejoría con cloxacilina deberían, además de retirarse los dispositivos vasculares cuando corresponda, considerar el uso de vancomicina ${ }^{30}$.

\section{Conclusión}

En el caso de sospecha de una sepsis tardía, la prescripción de agentes antibacterianos debe tener en cuenta varias situaciones importantes como: el cuadro clínico, los datos de vigilancia bacteriológica existentes del paciente y de la UCIN y la disposición de datos epidemiológicos en la literatura médica actual con respecto a la infección nosocomial neonatal. En estos casos, se necesita un enfoque racional con miras a la prescripción de una combinación de agentes antibacterianos con el espectro más reducido posible. Cloxacilina, en combinación con un aminoglucósido, es eficaz en la mayoría de los casos de infecciones por SCN que rara vez causan sepsis grave y casi siempre da tiempo al médico tratante para el cambio a vancomicina si la cepa identificada es resistente al tratamiento inicial. Incluso si hay una necesidad de cambiar, la mayoría de las veces se observa mejoría clínica y de los parámetros de laboratorio antes del cambio de tratamiento antimicrobiano ${ }^{32}$.
Más importante aún, este enfoque racional permite disminuir el uso excesivo de vancomicina, ya que, ésta presenta diversos problemas condicionados por múltiples factores tales como: la elevada variabilidad farmacocinética inter-individual e intra-individual en este grupo poblacional, la necesidad de uso de concentraciones elevadas de vancomicina lo que conlleva una mayor toxicidad y la relación entre el fracaso terapéutico con persistencia de concentraciones séricas inferiores a la $\mathrm{CIM}^{32}$. La optimización del tratamiento con vancomicina en RNPret mediante la monitorización de concentraciones plasmáticas es una práctica recomendada, pero que lleva a una constante toma de exámenes en una población de muy bajo peso y que frecuentemente tienen serias dificultades en alcanzar concentraciones terapéuticas adecuadas a corto plazo.

Basado en lo expuesto en esta revisión, estimamos el uso de cloxacilina como tratamiento empírico de una sepsis neonatal tardía por probable SCN en RNPret y RNT, ya que, de acuerdo a lo señalado, aún siendo estos agentes infecciosos resistentes a cloxacilina, no son de evolución invasora ni catastrófica y nos permite realizar el cambio a vancomicina al momento de obtenerse el antibiograma, siempre y cuando la evolución clínica del paciente no sea satisfactoria. Por otra parte, en muchas ocasiones se establecen tratamientos empírico iniciales por la sospecha de sepsis neonatal tardía, la cual termina por descartarse y se suspenden los antimicrobianos; mirado desde esta perspectiva, nos parece que la decisión de iniciar primeramente el tratamiento con cloxacilina podría disminuir el sobreuso de vancomicina y los problemas que esto conlleva.

Como último punto importante a considerar queremos recalcar y recomendar, que siendo éste un artículo de revisión, cada UCIN debe analizar y conocer su epidemiología local, además de tener claridad de su incidencia de SARM, ya que sólo así se podrá decidir el tratamiento empírico más adecuado a cada unidad.

Agradecimiento: A Isolda Budnik por su colaboración.

\section{Resumen}

Vancomicina se utiliza hace más de 50 años en unidades de cuidados intensivos neonatales (UCIN) como terapia de elección en sospecha de sepsis neonatal tardía; su principal indicación se fundamenta en que Staphylococcus coagulasa negativa (SCN) es el principal microorganismo que ocasiona sepsis tardía y éste es habitualmente resistente a cloxacilina; sin embargo, su virulencia es baja y la sepsis fulminante es inusual. Lamentablemente la prescripción de vancomicina se ha 
convertido en un grave problema en las UCIN, debido a diversas razones incluyendo: alta variabilidad farmacocinética del fármaco, dificultad en alcanzar concentraciones plasmáticas apropiadas y aumento de la concentración inhibitoria mínima (CIM), implicando además una mayor probabilidad de seleccionar cepas resistentes y aumento de otro tipo de infecciones ocasionadas por bacilos gramnegativos resistentes y candidiasis invasora.
Considerando lo anteriormente señalado y a lo publicado en la literatura médica con respecto a las infecciones en neonatología, debido a su comportamiento clínico diferente a hospederos en otras etapas de la vida, resulta de suma importancia replantear el uso de vancomicina basado en fundamentos teóricos que avalen la seguridad de no utilizar este antimicrobiano como primera línea en sepsis neonatal tardía.

\section{Referencias bibliográficas}

1.- Mehrotra N, Tang L, Phelps S J, Meibohm B. Evaluation of vancomycin dosing regimens in preterm and term neonates using Monte Carlo simulations. Pharmacotherapy 2012; 32 (5): 408-19.

2.- Hemels M A, van den Hoogen A, VerboonMaciolek M A, Fleer A, Krediet T G. A seven-year survey of management of coagulasenegative staphylococcal sepsis in the neonatal intensive care unit: vancomycin may not be necessary as empiric therapy. Neonatology 2011; 100 (2): 180-5.

3.- Lawrence S L, Roth V, Slinger R, Toye B, Gaboury I, Lemyre B. Cloxacillin versus vancomycin for presumed late-onset sepsis in the Neonatal Intensive Care Unit and the impact upon outcome of coagulase negative staphylococcal bacteremia: a retrospective cohort study. BMC Pediatr 2005; 5 (49): 1-8.

4.- Brilene T, Soeorg H, Kiis M, Sepp E, Kõljalg S, Lõivukene K. In vitro synergy of oxacillin and gentamicin against coagulasenegative staphylococci from blood cultures of neonates with late-onset sepsis. APMIS 2013; 121(9): 859-64

5.- Díaz M, Trigoso Agudo C, Damiani Moisés E, Ode Hiramatsu Y, Navía Bueno M. Agentes infecciosos en sepsis neonatal servicio de neonatología Hospital de la mujer-Inlasa. Cuad Hosp Clín 2005; 50 (1): 9-15.

6.- Genes L, Lacarrubba J, Mir R, Céspedes E, Mendieta E. Sepsis neonatal. Caracterización en recién nacidos de muy bajo peso. Experiencia de once años. Pediatr (Asunción) 2013; 40 (2): 145-54

7.- Tapia JL, Reichhard C, Saldías MI, Abarzúa F, Pérez ME, González A, et al. Sepsis neonatal en la era de profilaxis antimicrobiana prenatal. Rev Chilena Infectol 2007; 24 (2): 111-6.

8.- Otaíza F, Bustamante R, Sánchez P. Informe de Vigilancia de Infecciones Asociadas a la Atención de Salud 2012. Departamento de Calidad y Seguridad del paciente. Programa control de IAAS. MINSAL. Chile (pag 31-32).

9.- Jacqz-Aigrain E, Zhao W, Sharland M, van den Anker J N. Use of antibacterial agents in the neonate: 50 years of experience with vancomycin administration. Semin Fetal Neonatal Med 2013; 18 (1): 28-34.

10.- Wadw Kelly C, Benjamin Daniel K. Chapter 37: Clinical pharmacology of antiinfective drugs. Jack S. Remington, Jerome O. Klein, Christopher B. Wilson, Victor Nizet, Yvonne Maldonado. Infectious Diseases of the Fetus and Newborn Infant. 7th edition. Philadelphia: Elsevier Saunders; 2010, p. 1160-211.

11.- De Cock R F, Allegaert K, Brussee J M, Sherwin C M, Mulla H, de Hoog M, et al. Simultaneous pharmacokinetic modeling of gentamicin, tobramycin and vancomycin clearance from neonates to adults: towards a semi-physiological function for maturation in glomerular filtration. Pharm Res 2014; 31 (10): 2643-54

12.- Gwee A, Cranswick N, Metz D, Coghlan B, Daley A J, Bryant P A, et al. Neonatal vancomycin continuous infusion: still a confusion?. Pediatr Infect Dis J 2014; 33 (6): 600-5.

13.- Patel A D, Anand D, Lucas C, Thomson A H. Continuous infusion of vancomycin in neonates. Arch Dis Child 2013; 98 (6): 478-9.

14.- Zhao W, Lopez E, Biran V, Durrmeyer X, Fakhoury M, Jacqz-Aigrain E. Vancomycin continuous infusion in neonates: dosing optimisation and therapeutic drug monitoring. Arch Dis Child. 2013; 98 (6): 449- 53.

15.- Irikura M, Fujiyama A, Saita F, Fukushima S, Kitaoka H, Fukuda T, et al. Evaluation of the vancomycin dosage regimen based on serum creatinine used in the neonatal intensive care unit. Pediatr Int 2011; 53 (6): 1038-44.

16.- de Hoog M, Mouton J W, van den Anker J N. Vancomycin: pharmacokinetics and administration regimens in neonates. Clin Pharmacokinet 2004; 43 (7): 417-40.

17.- Sammons H M, Starkey E. Vancomycin use in neonates and children: evidence-based practice is needed. Arch Dis Child 2013; 98 (6): 447-8.

18.- Juan Aguilar M, Ferriols Lisart R, Tosca Segura R, Alós Almiñana M. Design and validation of a dosing algorithm for vancomycin in premature neonates. An Pediatr (Barc) 2008; 68 (2): $117-23$

19.- Allegaert K, van den Anker J N. Creatinine- based vancomycin dosing regimens in neonates: there is more to consider than the variation in drug assay. Pharmacotherapy 2012; 32 (9): e174-5.

20.- Plan O, Cambonie G, Barbotte E, Meyer P, Devine C, Milesi C, et al. Continuous-infusion vancomycin therapy for preterm neonates with suspected or documented Gram-positive infections: a new dosage schedule. Arch Dis Child Fetal Neonatal Ed 2008; 93 (6): F418-21.

21.- Ward R M, Allegaert K, de Groot R, van den Anker J N. Commentary: continuous infusion of vancomycin in neonates: to use or not to use remains the question. Pediatr Infect Dis J 2014; 33 (6): 606- 7.

22.- Lo Y L, van Hasselt J G, Heng S C, Lim C T, Lee T C, Charles B G. Population pharmacokinetics of vancomycin in premature Malaysian neonates: identification of predictors for dosing determination. Antimicrob Agents Chemother 2010; 54 (6): 2626- 32.

23.- Roberts J K, Stockmann C, Constance J E, Stiers J, Spigarelli M G, Ward R M, et al. Pharmacokinetics and pharmacodynamics of antibacterials, antifungals, and antivirals used most frequently in neonates and infants. Clin Pharmacokinet 2014; 53 (7): 581-610.

24.- Acuña C, Morales J, Castillo C, Torres J P. Pharmacokinetics of vancomycin in children hospitalized in a critical care unit. Rev Chilena Infectol 2013; 30 (6): 585-90.

25.- Torres J, Acuña C, Castillo C, Aravena D, Villarroel G, Morales J. Vancomycin pharmacokinetics in children admitted to the Pediatric (PICU) and Neonatal (NICU) Intensive Care Units. Interscience Conference on Antimicrobial Agents and ChemotherapyICAAC. September 10-13, 2013. Denver, Colorado, USA. G-991, página 138.

26.- Young T, Magnum B. Capítulo 1: Antibióticos. Neofax: Manual de drogas neonatológicas. 18 Edición en español. Editorial Panamericana; 2006, p. 81.

27.- Pacifici G M, Allegaert K. Clinical pharmacokinetics of vancomycin in the neonate: a review. Clinics (Sao Paulo) 2012; 67 (7): 831- 7.

28.- van den Hoogen A, Gerards L J, VerboonMaciolek M A, Fleer A, Krediet T G. Long-term 
trends in the epidemiology of neonatal sepsis and antibiotic susceptibility of causative agents. Neonatology 2010; 97 (1): 22-8.

29.- Chiu C H, Michelow I C, Cronin J, Ringer S A, Ferris T G, Puopolo K M. Effectiveness of a guideline to reduce vancomycin use in the neonatal intensive care unit. Pediatr Infect Dis J 2011; 30 (4): 273- 8.
30.- Blayney M P, Al Madani M. Coagulasenegative staphylococcal infections in a neonatal intensive care unit: In vivo response to cloxacillin. Paediatr Child Health 2006; 11 (10): 659-63.

31.- Fleer A, Hemels M A, Paauw A, Krediet T G. Reduced expression of PBP-2A by neonatal $m e c \mathrm{~A}$-positive coagulase-negative staphylococci
(CoNS) blood isolates: $\beta$-lactams are useful first-line agents for the treatment of neonatal CoNS sepsis, restricting the use of vancomycin. J Antimicrob Chemother 2012; 67 (7): 1616- 8 .

32.- van den Anker J N. How to optimize the evaluation and use of antibiotics in neonates. Early Hum Dev 2014; 90 Suppl 1: S10-2. 